\title{
Hepatische Amyloidose als seltene Differenzialdiagnose bei progredientem Leberversagen
}

Dominik Bettinger, Lisa Lutz, Michael Schultheiß, Martin Werner, Robert Thimme, Christoph Neumann-Haefelin

Ein 63-jähriger Mann mit anamnestisch moderatem Alkoholkonsum hat eine vergrößerte Leber, erhöhte Leberwerte und Aszites. Da die Ärzte eine Virushepatitis, Autoimmunhepatitis, primär biliäre Cholangitis und metabolisch-genetische Lebererkrankung ausschließen können, stellen sie die Verdachtsdiagnose einer akuten Alkoholhepatitis. Erst die histopathologische Untersuchung offenbart, woran der Patient leidet. Wenige Tage später stirbt er.

Seltene Erkrankung I Eine systemische Amyloidose ist eine seltene Erkrankung, welche durch extrazelluläre Ablagerungen von unlöslichen Fibrillen gekennzeichnet ist. Dabei unterscheidet man:

- Leichtkettenamyloidose (AL-Amyloidose, primäre Amyloidose)

- Amyloid-Amyloidose (AA-Amyloidose, sekundäre Amyloidose), sowie seltenere Formen wie

- $\beta 2$-Mikroglobulin-assoziierte Form und

- familiäre Amyloidose-Typen (TransthyretinAmyloidose)

Amyloidablagerungen findet man am häufigsten in der Niere, dem Herzen, in den peripheren Nerven sowie in bis zu 70\% der Patienten auch in der Leber [2]. Eine Leberbeteiligung ist klinisch oft inapparent, kann sich aber auch in einem Leberversagen manifestieren $[3,5,6]$.

\section{Kasuistik}

Anamnese I Bei einem 63-jährigen Mann tritt erstmals ein Aszites auf. Er wird von seinem Hausarzt in unser Leberzentrum eingewiesen. Er leidet zudem unter einem leichten Druckschmerz im rechten oberen Quadranten des Abdomens. Bevor der Aszites auftrat, verlor er ungewollt $4 \mathrm{~kg}$ Körpergewicht innerhalb von 3 Wochen. Der Patient beklagt Appetitlosigkeit, Abgeschlagenheit und einen Leistungsknick. Fieber und weitere Symptome verneint er. Die medizinische Vorgeschichte ist bis auf eine bestehende benigne Prostatahyperplasie und eine leichte Hypercholesterinämie unauffällig. Auf Nachfrage liegt ein fraglich moderater Alkoholkonsum vor. Des Weiteren lässt sich ein Ex-Nikotinabusus (kumulativ 15 pack years) eruieren. Der Mann gibt an, dass in seiner Familie keine kardiovaskulären, neoplastischen und gastroenterologischhepatologischen Erkrankungen vorliegen.

Körperliche Untersuchung I Der Patient befindet sich in einem reduzierten Allgemein- und normalen Ernährungszustand. In der klinischen Untersuchung des Abdomens ist die Leber ver- größert tastbar und es lässt sich Aszites nachweisen. Es zeigen sich zudem leichte periphere Ödeme. Die Untersuchungen des Herzens, der Lunge und die neurologische Untersuchung ergeben keine pathologischen Befunde.

Laborbefunde I Die Transaminasen sind mäßig erhöht (AST 142 U/l, ALT 67U/1), die alkalische Phosphatase liegt bei $1142 \mathrm{U} / \mathrm{l}$, das Bilirubin bei $4,1 \mathrm{mg} / \mathrm{dl}$ mit einem direkten Anteil von $2,1 \mathrm{mg} / \mathrm{dl}$. Die $\gamma$-GT ist erhöht $(818 \mathrm{U} / \mathrm{l})$. Es lässt sich eine normale plasmatische Gerinnung mit einem INR von 1,17 nachweisen. Das Blutbild zeigt einen Hämoglobinwert von $17,1 \mathrm{~g} / \mathrm{dl}$, normwertige Thrombozyten $\left(153 \times 10^{9} / \mu \mathrm{l}\right)$ und normwertige Leukozyten $\left(6,09 \times 10^{9} / \mu \mathrm{l}\right)$.

Untersuchung des Aszitespunktats I Es erfolgt eine diagnostische und therapeutische Punktion des Aszites. Darin lassen sich keine inflammatorischen oder malignen Zellen nachweisen. Insgesamt ist der Serum-Aszites-Albumin-Gradient niedrig $(S A A G<1,1 \mathrm{~g} / \mathrm{dl}$ ).

Abdomensonografie I Hier zeigt sich ein 4-Quadranten-Aszites. Sichere Hinweise auf eine Leberzirrhose gibt es nicht. Des Weiteren kann eine intra- und extrahepatische Cholestase ausgeschlossen werden. Duplexsonografisch ergeben sich keine Hinweise auf eine Pfortaderthrombose oder ein Budd-Chiari-Syndrom. In weiteren serologischen, virologischen und immunologischen Untersuchungen können eine Virushepatitis, eine Autoimmunhepatitis, eine primär biliäre Cholangitis sowie metabolisch-genetische Lebererkrankungen (Hämochromatose, alpha-1-Antitrypsinmangel und M. Wilson) ausgeschlossen werden. Zusammenfassend ergibt sich bei Ausschluss der oben genannten Ursachen und bei anamnestisch bestehendem fraglich moderatem Alkoholkonsum der Verdacht auf eine akute Alkoholhepatitis.

Weiterführende hepatologische Diagnostik I In den folgenden Wochen verschlechtern sich die Leber- und Nierenfunktion rapide (maximales Bilirubin 34,5 mg/dl, maximale ALT 428 U/1, ma- 
Abb. 1 Histologische Untersuchung des Leberbiopsates mit einer HE-Färbung (A, B), Kongorotfärbung $(C)$ sowie eine immunhistochemische Färbung auf kappa- (D) und lambda-Leichtketten (E). Die Sterne in B zeigen komprimierte und atrophische Hepatozyten.

Orginalvergrößerungen: $20 \times(A), 200 \times(B, C, D, E)$

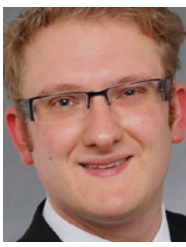

Dr. med. Dominik Bettinger ist Assistenzarzt an der Klinik für Innere Medizin II, Universitätsklinikum Freiburg.

dominik.bettinger@ uniklinik-freiburg.de

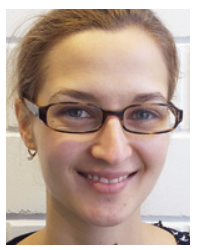

Dr. med. Lisa Lutz ist Assistenzärztin am Institut für Klinische Pathologie, Universitätsklinikum Freiburg.

lisa.lutz@

uniklinik-freiburg.de

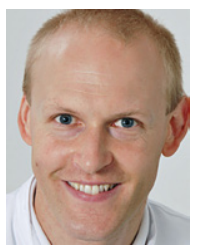

Dr. med. Michael Schultheiß ist Oberarzt an der Klinik für Innere Medizin II, Universitätsklinikum Freiburg. michael.schultheiss@ uniklinik-freiburg.de
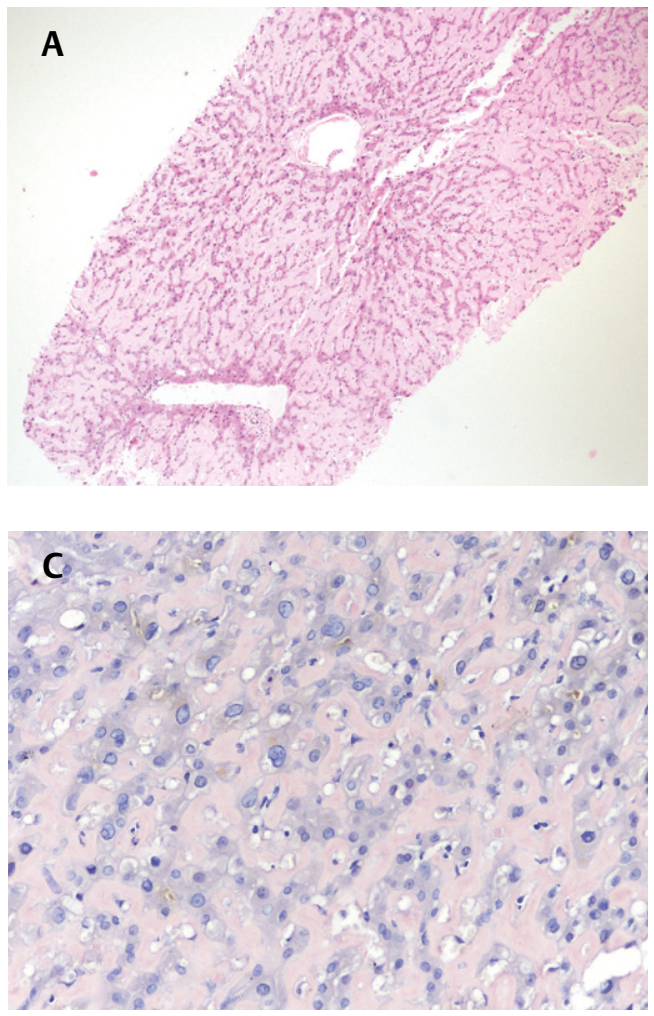

ximale AST 659U/1, maximaler INR 2,81, maximales Serumkreatinin $9,06 \mathrm{mg} / \mathrm{dl}$ ). Daher entscheiden wir uns zur Durchführung einer transjugulären Leberbiopsie. Diese wird komplikationslos durchgeführt, insbesondere ohne postinterventionelle Blutungskomplikationen.

Histopathologische Untersuchung I Das Biopsat enthält homogene, amorphe und eosinophile $\mathrm{Ab}$ lagerungen im Disse-Raum ( $\bullet$ Abb. $1 \mathrm{~A}$ und B), die sich in der Congo-Rot-Färbung anfärben lassen ( $\wedge$ Abb. 1C). Die weiteren immunhistochemischen Färbungen zeigen eine positive Färbung für kappa-Leichtketten ( $\bullet$ Abb. 1D), während sich keine lambda-Leichtketten nachweisen lassen ( Abb. 1E). In Zusammenschau dieser Befunde wird die Diagnose einer AL-Amyloidose vom Typ kappa gestellt.

Weiterer Verlauf I Als Hinweis auf eine Nierenbeteiligung lässt sich eine Proteinurie nachweisen. In der durchgeführten echokardiografischen Untersuchung zeigt sich eine diastolische Dysfunktion als Hinweis auf eine restriktive Kardiomyopathie, was als typische Manifestation einer kardialen Amyloidose beschrieben ist. Da die Erkrankung schnell fortschreitet, wird sofort eine Chemotherapie mit Dexamethason, Cyclophosphamid und Bortezomib eingeleitet. Innerhalb von wenigen Tagen tritt jedoch Fieber auf. Aufgrund einer Streptococcus-pneumoniae-Bakteriämie muss die Chemotherapie gestoppt werden. Die komplizierende Infektion führt zu einem zunehmenden Leber- und Nierenversagen, woran der Patient schließlich stirbt.
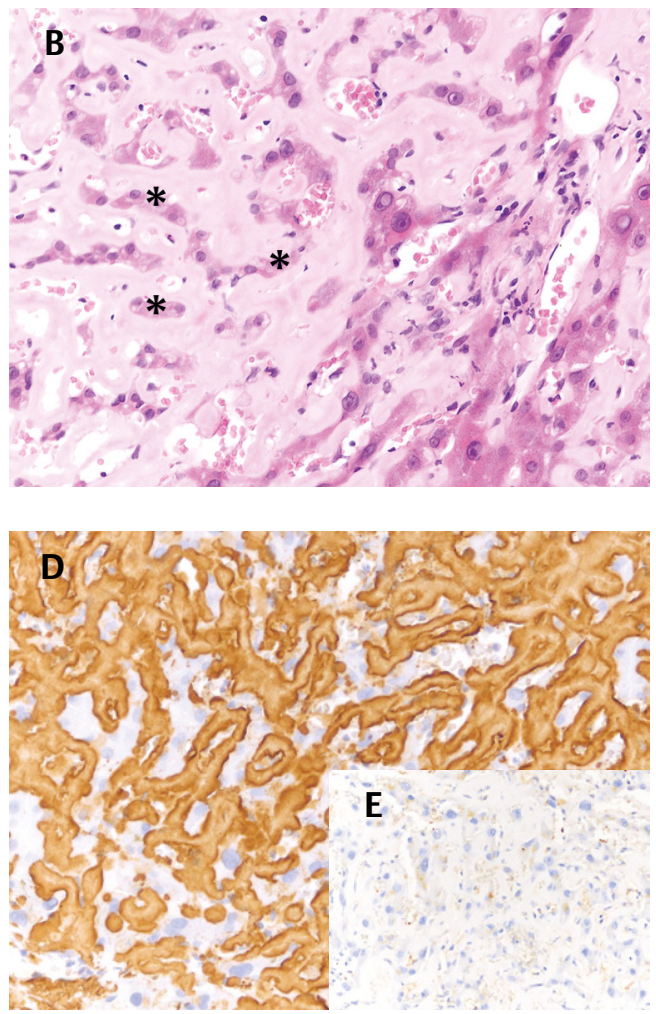

\section{Diskussion}

Selten gestellte Differenzialdiagnose | Eine Ablagerung von Amyloid in der Leber und Milz kommt bei einer systemischen Amyloidose häufig vor, ohne jedoch Symptome zu verursachen. Wenn sie auftreten, so sind diese in den meisten Fällen unspezifisch. Daher wird eine Amyloidose selten als mögliche Differenzialdiagnose berücksichtigt ( Tab. 1). Eine Hepatomegalie findet sich bei $24-33 \%$ der Patienten $[7,8]$. Sie ist jedoch nicht pathognomonisch für eine Leberbeteiligung, da in ca. 33\% der Patienten mit einer primären systemischen Amyloidose eine Hepatomegalie vorliegen kann, ohne dass eine direkte hepatische Manifestation vorliegt [3, 9, 10]. Ist die Leber beteiligt, lässt sich bei $80-90 \%$ der Patienten auch eine Hepatomegalie nachweisen [11].

Laborparameter oft nicht wegweisend | Pathologische Leberwerte, vor allem erhöhte Werte der alkalischen Phosphatase und der AST, findet man vor allem bei einer fortgeschrittenen Leberbeteiligung [4]. Ein erhöhtes Bilirubin weist auf einen Progress der Erkrankung mit einer negativen Prognose hin [4]. Die Einschränkung der Leberfunktion kann jedoch zum einen durch die hepatische Beteiligung der Amyloidose bedingt sein, zum anderen aber auch durch eine Stauungshepatopathie bei einer Amyloid-induzierten Herzinsuffizienz [12].

Die Leberwerte sind wenig sensitiv und spezifisch für die Diagnose einer Amyloidose. 


Symptome
- Ungewollter Gewichtsverlust (72\%)
- Fatigue (60\%)
- Bauchschmerzen (53\%)
- Übelkeit (15\%)

$$
\begin{aligned}
& \text { Symptome } \\
& \text { - Ungewollter Ge } \\
& \text { - Fatigue (60\%) } \\
& \text { - Bauchschmerze } \\
& \text { - Übelkeit (15\%) }
\end{aligned}
$$

\author{
Klinische Untersuchung \\ - Hepatomegalie (81\%) \\ - Splenomegalie (10\%) \\ - Aszites (42\%) \\ - Purpura (15\%) \\ - Spider naevi (7\%) \\ - Ödeme $(26 \%)$
}

Leberbiopsie sichert Diagnose | Aus diesem Grund ist es oft nur mit Hilfe einer Leberbiopsie möglich die Diagnose zu stellen [13, 14]. Untersuchungen zeigten, dass nur wenige Ärzte eine Amyloidose als Diagnose vermuteten, bevor eine Leberbiopsie durchgeführt wurde, was sich auch in unserem Fall wiederspiegelt [4]. Eine Leberbiopsie kann entweder perkutan oder transjugulär durchgeführt werden. Blutungskomplikationen (insbesondere eine Leberblutung als schwerwiegendste Komplikation) bei einer perkutanen Punktion sind zwar allgemein selten, jedoch ist das Risiko bei Patienten mit Gerinnungsanomalitäten, wie man sie unter anderem auch bei der Amyloidose beobachtet, signifikant erhöht [4]. Eine transjuguläre Leberbiopsie hingegen reduziert das Risiko für Blutungen, da die Leberkapsel nicht perforiert wird. Zudem stellt das Vorliegen von Aszites eine Kontraindikation für die Durchführung einer perkutanen Leberbiopsie dar, sodass aufgrund der Gerinnungsstörung und des vorliegenden Aszites in unserem Fall eine transjuguläre Biopsie durchgeführt wurde [15].

Therapie I Die Behandlung hängt vom Typ der Amyloidose und von der Organbeteiligung ab. Das Ziel der Therapie der AL-Amyloidose ist eine Eradikation der monoklonalen Plasmazellen, die unkontrolliert freie Leichtketten produzieren und somit zu einer Ablagerung in den Organen führen. Ein weiteres Ziel der Therapie ist aber auch die Organfunktionen durch eine supportive Therapie zu erhalten [17]. Melphalan, Dexamethason und Cyclophosphamid sowie Prednisolon und seit einigen Jahren auch Bortezomib sind häufige Substanzen, die in der Therapie der ALAmyloidose eingesetzt werden [17-19]. Des Weiteren wurde in selektionierten Patienten der Nutzen einer Hochdosischemotherapie mit anschließender autologer peripherer Stammzelltransplantation untersucht. Hierbei zeigte sich bei ausgewählten Patienten ein verbessertes Überleben [17]. Jedoch ist die Therapie der Amyloidose oft durch eine verzögerte Diagnosesicherung und durch eine schnelle Krankheitsprogression limitiert und zudem ist eine aggressive Therapie aufgrund einer Leber- und Niereninsuffizienz kaum möglich.

Prognose | Aufgrund eines schnellen Fortschreitens der Erkrankung mit einer progredienten Verschlechterung der Funktion der betroffenen Organe, ist die Prognose mit einem medianen
Gesamtüberleben von 8,5 Monaten bei einer bestehenden Leberbeteiligung sehr schlecht [4]. Erhöhte Bilirubinwerte, eine bestehende Herzinsuffizienz und erhöhte Thrombozyten sind negative prognostische Faktoren für das Gesamtüberleben.

\section{Konsequenz für Klinik und Praxis}

- Eine hepatische Amyloidose wird meist zu spät diagnostiziert. Da die Erkrankung schnell fortschreitet, ist die Prognose der Betroffenen schlecht.

- Die klinischen, laborchemischen und bildmorphologischen Veränderungen sind unspezifisch, weshalb erst über die Leberhistologie die Diagnose gelingt.

- Eine Leberhistologie sollte daher bei allen unklaren akuten und chronischen Hepatopathien bei sonst unauffälliger Abklärung in der Diagnostik erwogen werden.

\section{Literatur}

1 Monzawa S, Tsukamoto T, Omata K et al. A case with primary amyloidosis of the liver and spleen: radiologic findings. Eur J Radiol 2002; 41: 237-241

2 Buck FS, Koss MN. Hepatic amyloidosis: morphologic differences between systemic AL and AA types. Hum Pathol 1991; 22: 904-907

3 Gertz MA, Kyle RA. Hepatic amyloidosis (primary [AL], immunoglobulin light chain): the natural history in 80 patients. Am J Med 1988; 85: 73-80

4 Park MA, Mueller PS, Kyle RA et al. Primary (AL) hepatic amyloidosis: clinical features and natural history in 98 patients. Medicine 2003; 82: 291-298

5 Kitamura Y, Yokomori H. Hepatic amyloidosis manifesting severe intrahepatic cholestasis. Intern Med 2005; 44: 675-676

6 Giers K, Niemczyk S, Ziarkiewicz-Wroblewska B et al. A rare cause of acute hepatic failure. Liver Int 2008; 28: 1478-3231

7 Kyle RA, Greipp PR. Amyloidosis (AL). Clinical and laboratory features in 229 cases. Mayo Clin Proc 1983; 58: 665-683

8 Kyle RA, Gertz MA. Primary systemic amyloidosis: clinical and laboratory features in 474 cases. Semin Hematol 1995; 32: 45-59

9 Levy M, Polliack A, Lender M et al. The liver in amyloidosis. Digestion 1974; 10: 40-51

10 Levine RA. Amyloid disease of the liver. Correlation of clinical, functional and morphologic features in forty-seven patients. Am J Med 1962; 33: 349-357

11 Gertz MA, Kyle RA. Hepatic amyloidosis: clinical appraisal in 77 patients. Hepatology 1997; 25: 118-121

Vollständiges Literaturverzeichnis unter http://dx.doi.org/10.1055/s-0042-113053
Tab. 1 Symptome und Befunde der klinischen Untersuchung bei Patienten mit einer Leberbeteiligung bei einer Amyloidose [4].

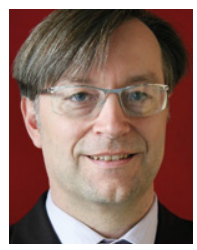

Prof. Dr. med.

Martin Werner

ist Direktor des Instituts für Klinische Pathologie, Universitätsklinikum Freiburg.

martin.werner@

uniklinik-freiburg.de

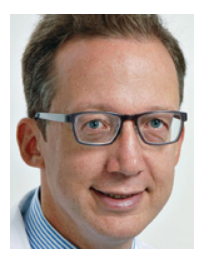

Prof. Dr. med.

Robert Thimme

ist ärztlicher Direktor der Klinik für Innere Medizin II, Universitätsklinikum Freiburg.

robert.thimme@

uniklinik-freiburg.de

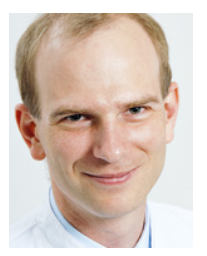

PD Dr. med. Christoph Neumann-Haefelin ist Oberarzt an der Klinik für Innere Medizin II, Universitätsklinikum Freiburg. christoph.neumannhaefelin@

uniklinik-freiburg.de

Interessenkonflikt

Die Autoren geben an, dass kein Interessenkonflikt besteht.

DOI 10.1055/s-0042-113053

Dtsch Med Wochenschr 2015; 140: 1387-1389 (c) Georg Thieme Verlag KG . Stuttgart . New York . ISSN 0012-0472 
12 Richman SM, Delman AJ, Grob D. Alterations in indices of liver function in congestive heart failure with particular reference to serum enzymes. Am J Med 1961; 30: 211-225

13 Gertz MA. Immunoglobulin light chain amyloidosis: 2014 update on diagnosis, prognosis, and treatment. Am J Hematol 2014; 89: 1132-1140

14 Chee CE, Lacy MQ, Dogan A et al. Pitfalls in the diagnosis of primary amyloidosis. Clin Lymphoma Myeloma Leuk 2010; 10: 177-180

15 Dohan A, Guerrache Y, Boudiaf M et al. Transjugular liver biopsy: indications, technique and results. Diagn Interv Imaging 2014; 95: 11-15

16 Cholongitas E, Burroughs AK. Liver: Transjugular liver biopsy yields high-quality samples. Nat Rev Gastroenterol Hepatol 2012; 9: 491-492

17 Mahmood S, Palladini G, Sanchorawala V et al. Update on treatment of light chain amyloidosis. Haematologica 2014; 99: 209-221

18 Kyle RA, Gertz MA, Greipp PR et al. Long-term survival (10 years or more) in 30 patients with primary amyloidosis. Blood 1999; 93: 1062-1066

19 Reece DE, Hegenbart U, Sanchorawala V et al. Efficacy and safety of once-weekly and twiceweekly bortezomib in patients with relapsed systemic AL amyloidosis: results of a phase $1 / 2$ study. Blood 2011; 118: 865-873 